\title{
Transarterial Embolization for Cervical Hemangioma Associated With Kasabach-Merritt Syndrome -Case Report-
}

\author{
Yukiko ENOMOTO, ${ }^{1}$ Shinichi YOSHIMURA, ${ }^{1}$ \\ Yusuke EGASHIRA, ${ }^{1}$ and Toru IWAMA ${ }^{1}$ \\ ${ }^{1}$ Department of Neurosurgery, Gifu University Graduate School of Medicine, Gifu
}

\begin{abstract}
A 2-month-old girl presented with cervical hemangioma associated with Kasabach-Merritt syndrome. The patient had previously undergone systemic administration of corticosteroids (prednisolone 2 $\mathrm{mg} / \mathrm{kg} / \mathrm{day})$ and radiotherapy (0.5 Gy $\times 5$ times), which temporarily increased the platelet count, but the effects were short-lived and thrombocytopenia gradually worsened again despite increased dosage of prednisolone to $5 \mathrm{mg} / \mathrm{kg} / \mathrm{day}$. Computed tomography and magnetic resonance imaging revealed a hemangioma in the left retroauricular-cervical region with a well-enhanced, ill-defined margin and bony structure involvement. Diagnostic and therapeutic angiography were carried out through the femoral route under general anesthesia. Polyvinyl alcohol particles (250-350 $\mu \mathrm{m})$ suspended in contrast medium were slowly injected and a fibered coil was finally placed in the proximal portion of the arterial feeder. Angiography after embolization revealed a marked reduction in the tumor stain. Platelet count rapidly increased and reached the normal range on postembolization day 7 . Tumor tension decreased on the day after the procedure and tumor size gradually decreased. The patient was discharged with oral corticosteroid therapy at 6 months postembolization. Corticosteroid dose was gradually decreased and coagulation parameters were normal at 1 year postembolization.
\end{abstract}

Key words: hemangioma, Kasabach-Merritt syndrome, thrombocytopenia, infant, embolization

\section{Introduction}

Most vascular tumors occurring in infancy are clinically benign, such as infantile hemangioma, which grows rapidly at first and involutes spontaneously during childhood. However, some vascular tumors cause life-threatening thrombocytopenia in infants in a condition known as

Received August 3, 2010; Accepted October 12, 2010
Kasabach-Merritt syndrome (KMS). ${ }^{7)}$ Here we describe an infant with cervical hemangioma associated with KMS successfully treated by transarterial embolization.

\section{Case Presentation}

A 2-month-old girl was referred to our hospital because of thrombocytopenia and cervical hemangioma diagnosed by fetal ultrasonography at 36 weeks gestation. She was 


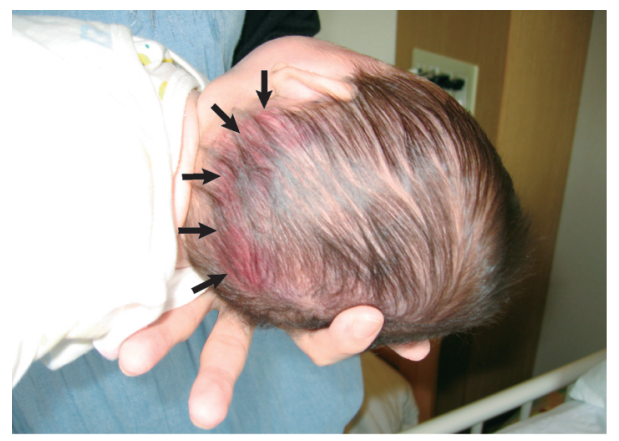

Fig. 1 Photograph showing hemangioma with purpura (arrows) on the neck.

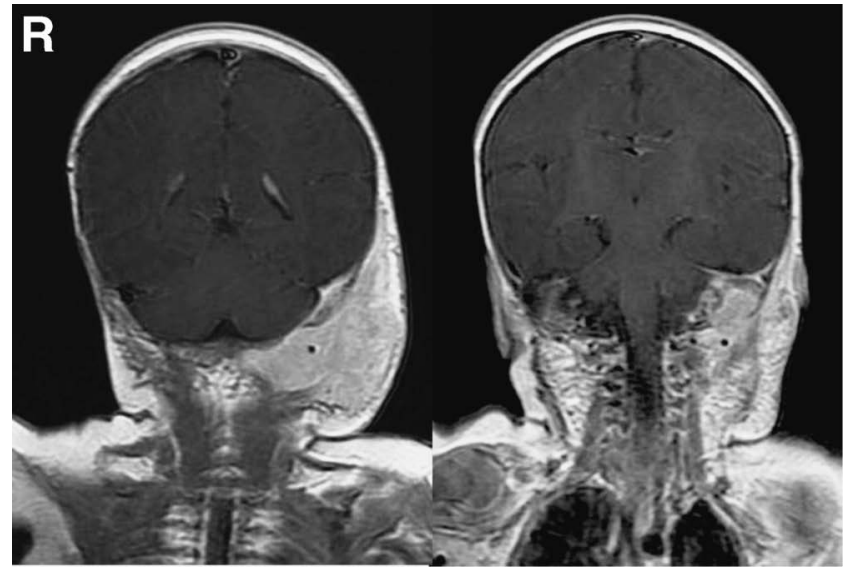

Fig. $2 T_{1}$-weighted magnetic resonance images with gadolinium revealing a huge hemangioma in the left retroauricular-cervical region.

born by scheduled caesarean section at 39 weeks (birth weight $2816 \mathrm{~g}$ ). At birth, a $6 \times 10 \mathrm{~cm}$ hard mass with purpura was noted in the left retroauricular-cervical region (Fig. 1). Laboratory tests revealed: platelet count $1.0 \times 10^{4}$ $/ \mu \mathrm{l}$, fibrinogen $70 \mathrm{mg} / \mathrm{dl}$, and fibrinogen degradation products $48.7 \mu \mathrm{g} / \mathrm{ml}$. Coagulation parameters were consistent with KMS. The patient had previously undergone systemic administration of corticosteroids (prednisolone 2 $\mathrm{mg} / \mathrm{kg} /$ day) and radiotherapy $(0.5 \mathrm{~Gy} \times 5$ times $)$. These treatments temporarily increased the platelet count, but the effects were short-lived and thrombocytopenia gradually worsened again although the dosage of prednisolone had been increased gradually up to $5 \mathrm{mg} / \mathrm{kg} / \mathrm{day}$. The patient was referred to our hospital for arterial embolization therapy at age 2 months. Computed tomography and magnetic resonance imaging revealed a hemangioma in the left retroauricular-cervical region with a well-enhanced, ill-defined margin and bony structure involvement (Fig. 2).

Diagnostic and therapeutic angiography were carried out through the femoral route under general anesthesia. A 4-French sheath was inserted percutaneously into the right femoral artery and a 4-French single lumen end-hole

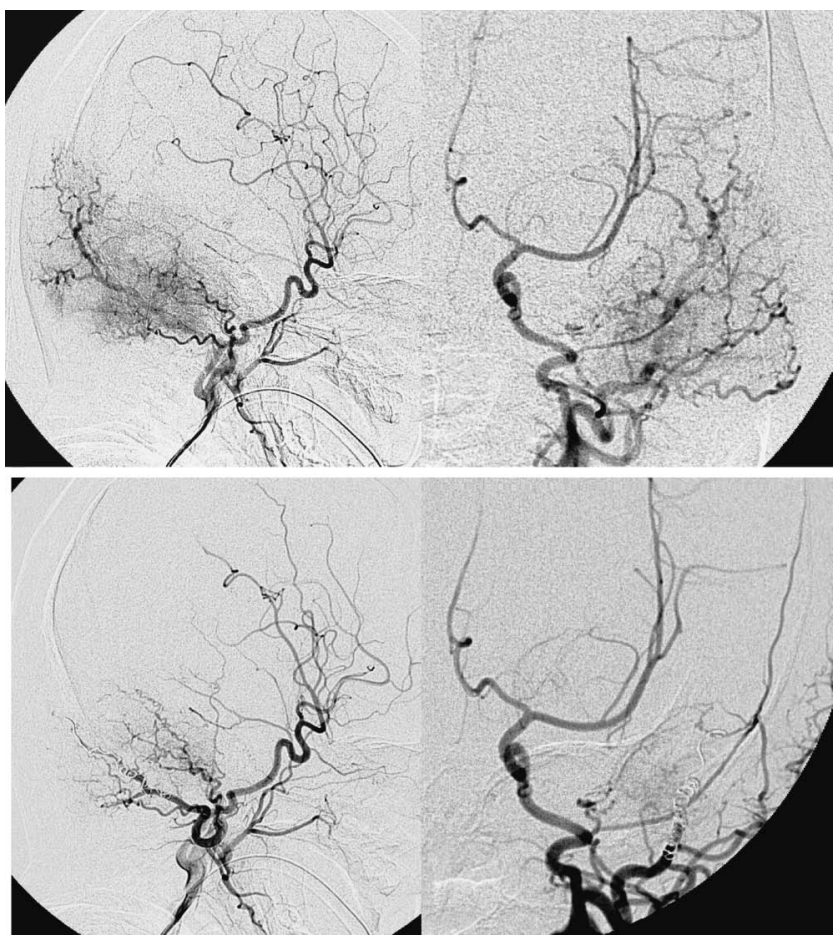

Fig. 3 Upper row: Left common carotid angiograms showing a tumor stain supplied from the left occipital artery and posterior auricular artery. Lower row: Post-embolization angiograms demonstrating marked reduction in the tumor stain.

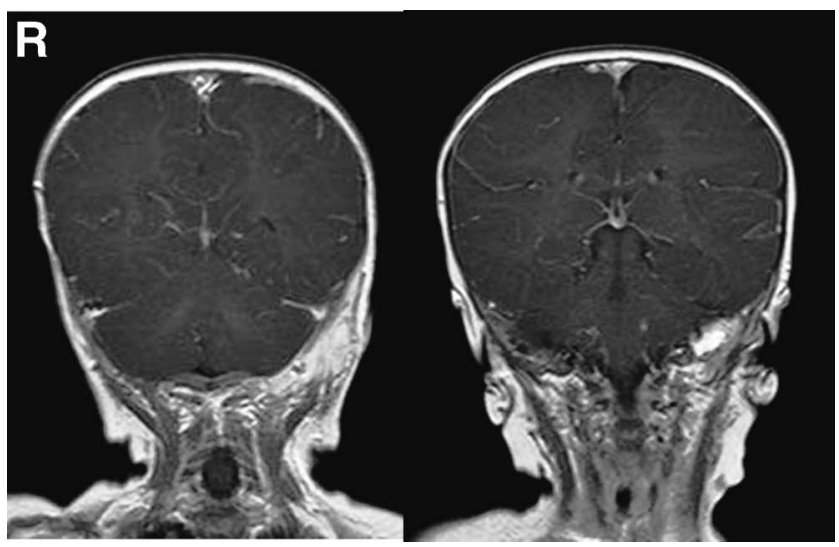

Fig. $4 T_{1}$-weighted magnetic resonance images with gadolinium obtained at 1-month follow up showing significant reduction in tumor size.

catheter was introduced into the left common carotid artery. No arteriovenous shunting was observed. Left carotid angiography showed that the occipital artery and the posterior auricular artery, but not the internal carotid system, supplied the hypervascular tumor. Using the 4French diagnostic catheter as a guiding catheter, a microcatheter (Renegade 18; Boston Scientific, Fremont, California, USA) was selectively positioned in the left occipital artery, which was the main arterial supply. Poly- 
vinyl alcohol (PVA) particles (250-350 $\mu \mathrm{m})$ were suspended in contrast medium, and the PVA suspension was slowly injected until the contrast medium reached the dose limit of $3 \mathrm{ml} / \mathrm{kg}$. A fibered coil (GDC-18 Vortex; Boston Scientific) was finally placed in the proximal portion of the arterial feeder, expecting a further decrease in residual tumor stain. Angiography after embolization revealed a marked reduction in the tumor stain (Fig. 3).

Platelet count rapidly increased and reached the normal range on postembolization day 7. Tumor tension decreased on the day after the procedure and tumor size gradually decreased (Fig. 4). The patient was discharged with oral corticosteroid therapy at 6 months postembolization. Corticosteroid dose was gradually decreased and coagulation parameters were normal at 1 year postembolization.

\section{Discussion}

Hemangioma in the leg extending into the trunk associated with thrombocytopenia in an infant (KMS) was first described by Kasabach and Merritt in 1940.7) The mortality rate of hemangioma with thrombocytopenia (i.e., KMS) is $12 \%$ to $24 \%$ resulting from hemorrhage, infection, invasion of vital structures, and multiple organ failure. ${ }^{2,10)}$ The goal of treating hemangioma as part of KMS is to control the thrombocytopenia and avoid the lethal complications. Several therapeutic options are available. Pharmacological treatments include systemic corticosteroid therapy, interferon therapy, anti-cancer drugs, and anti-platelet therapy, but the effectiveness of these treatments has been inconsistent. The most cost-effective treatment is highdose corticosteroid therapy. High-dose prednisolone (2 $\mathrm{mg} / \mathrm{kg} /$ day) therapy was successfully used in one case of KMS, ${ }^{9}$ but was ineffective in many cases. ${ }^{8,10,12,13)}$ The response rate to high-dose prednisone $(3-8 \mathrm{mg} / \mathrm{kg} /$ day) therapy among 11 cases of complex hemangioma was $45 \%$, and the cure rate was $18 \% .{ }^{14)}$ Interferon $\alpha 2 \mathrm{~b}$ therapy remains controversial, with a response rate of about $50 \%$. ${ }^{3,10)}$ Anti-cancer drugs, such as vincristine, are another treatment option. All 15 patients with KMS showed increased platelet counts and 13 patients had significant decreases in the tumor size after treatment with vincristine, but complications included abdominal pain, irritability, and transient loss of deep tendon reflexes. ${ }^{4)}$ Heparin and anti-platelet therapy have been used with varied success. ${ }^{2)}$ When conventional pharmacological treatments fail, radiation therapy, surgical resection, and transarterial embolization can be considered. Radiation therapy can obtain immediate increase in platelet count, but the effects are temporary and there is potential risk of radiation-induced malignancy. ${ }^{5}$ S Surgical resection is the definitive treatment for KMS, but the patient must be hematologically stable. ${ }^{1)}$ Transarterial embolization is very effective, safe, and an excellent therapeutic option. Successful embolization has been reported with several embolic materials, such as coils, ${ }^{6)}$ PVA, ${ }^{8,11,12)}$ and onyx. ${ }^{15)}$

We achieved an excellent outcome on our patient using transarterial embolization with PVA, which improved the hematological parameters dramatically. In this case, previ- ous treatments using systemic corticosteroid administration and radiation therapy were temporarily effective, but the thrombocytopenia gradually worsened again. Her parents rejected additional pharmacological treatment because of the possibility of side effects. Embolization of the vascular beds associated with platelet consumption appears to reduce localized coagulopathy associated with KMS. We decided to use PVA particles of $250-350 \mu \mathrm{m}$ size which can penetrate deep into the tumor and obliterate the vascular beds. Hemangioma associated with KMS is either Kaposiform hemangioendothelioma or tufted angioma that should be distinguished from infantile hemangioma. ${ }^{2,10,16)}$ Although the tumor was not identified histologically, magnetic resonance imaging demonstrated the characteristic findings of Kaposiform hemangioendothelioma or tufted angioma. ${ }^{10)}$

The present case of cervical hemangioma associated with KMS was successfully treated by transarterial embolization. Transarterial embolization is an effective therapeutic option for hemangioma associated with KMS if pharmacological treatments fail.

\section{References}

1) Drolet BA, Scott LA, Esterly NB, Gosain AK: Early surgical intervention in a patient with Kasabach-Merritt phenomenon. J Pediatr 138: 756-758, 2001

2) Enjolras O, Wassef M, Mazoyer E, Frieden IJ, Rieu PN, Drouet L, Taïeb A, Stalder JF, Escande JP: Infants with Kasabach-Merritt syndrome do not have "true" hemangiomas. J Pediatr 130: 631-640, 1997

3) Ezekowitz RA, Mulliken JB, Folkman J: Interferon alfa-2a therapy for life-threatening hemangiomas of infancy. N Engl J Med 326: 1456-1463, 1992

4) Haisley-Royster C, Enjolras O, Frieden IJ, Garzon M, Lee M, Oranje A, de Laat PC, Madern GC, Gonzalez F, Frangoul H, Le Moine P, Prose NS, Adams DM: Kasabach-merritt phenomenon: a retrospective study of treatment with vincristine. J Pediatr Hematol Oncol 24: 459-462, 2002

5) Imafuku S, Hosokawa C, Moroi Y, Furue M: Kasabach-Merritt syndrome associated with angiosarcoma of the scalp successfully treated with chemoradiotherapy. Acta Derm Venereol 88: 193-194, 2008

6) Jianhong L, Xianliang H, Xuewu J: Transcatheter arterial embolization in the treatment of extensive maxillofacial hemangioma in children. World J Surg 29: 1550-1556, 2005

7) Kasabach KK, Merritt HH: Capillary hemangioma with extensive purpura: Report of a case. Am J Dis Child 59: 1063-1070, 1940

8) Komiyama M, Nakajima H, Kitano S, Sakamoto H, Kurimasa $\mathrm{H}$, Ozaki H: Endovascular treatment of huge cervicofacial hemangioma complicated by Kasabach-Merritt syndrome. Pediatr Neurosurg 33: 26-30, 2000

9) Munn SE, Jackson JE, Jones RR: Tufted haemangioma responding to high-dose systemic steroids: a case report and review of the literature. Clin Exp Dermatol 19: 511-514, 1994

10) Sarkar M, Mulliken JB, Kozakewich HP, Robertson RL, Burrows PE: Thrombocytopenic coagulopathy (Kasabach-Merritt phenomenon) is associated with Kaposiform hemangioendothelioma and not with common infantile hemangioma. Plast Reconstr Surg 100: 1377-1386, 1997

11) Sato Y, Frey EE, Wicklund B, Kisker CT, Smith WL: Embolization therapy in the management of infantile hemangioma 
with Kasabach Merritt syndrome. Pediatr Radiol 17: 503-504, 1987

12) Stanley P, Gomperts E, Woolley MM: Kasabach-Merritt syndrome treated by therapeutic embolization with polyvinyl alcohol. Am J Pediatr Hematol Oncol 8: 308-311, 1986

13) Teillac-Hamel D, De Prost Y, Bodemer C, Andry P, Enjolras O, Sebag G, Brunelle F, Hubert P, Nihoul-Fekete C: Serious childhood angiomas: unsuccessful alpha-2b interferon treatment. A report of four cases. Br J Dermatol 129: 473-476, 1993

14) Weber TR, Connors RH, Tracy TF Jr, Bailey PV: Complex hemangiomas of infants and children. Individualized management in 22 cases. Arch Surg 125: 1017-1020, 1990

15) Wolfe SQ, Farhat H, Elhammady MS, Moftakhar R, Aziz-
Sultan MA: Transarterial embolization of a scalp hemangioma presenting with Kasabach-Merritt syndrome. J Neurosurg Pediatr 4: 453-457, 2009

16) Zukerberg LR, Nickoloff BJ, Weiss SW: Kaposiform hemangioendothelioma of infancy and childhood. An aggressive neoplasm associated with Kasabach-Merritt syndrome and lymphangiomatosis. Am J Surg Pathol 17: 321-328, 1993

Address reprint requests to: Shinichi Yoshimura, MD, PhD, Department of Neurosurgery, Gifu University Graduate School of Medicine, 1-1 Yanagido, Gifu 501-1194, Japan. e-mail:nohgeka@gifu-u.ac.jp 